\title{
Multiple stimuli responsive cyclodextrin based smart materials for drug delivery: a review
}

\author{
Sandhya Sadanandan *, Devika V, Niranjana Rajeev, Sreelekshmi P J, Amrutha Chandran, Goutami G B, and Aiswarya \\ Lakshmi S
}

Department of Chemistry, Amrita School of Arts and Sciences, Amrita Vishwa Vidyapeetham, Amritapuri-690525, India

\begin{abstract}
In this review we discuss about the research advances in multiple stimuli responsive cyclodextrin (CD) based smart materials. CD based systems which are responsive to temperature, $\mathrm{pH}$, enzymes, light, redox etc. along with various applications of these systems in the biomedical area are extensively covered. Current challenges and future perspectives of these smart stimuli-responsive drug delivery systems are also discussed.
\end{abstract}

\section{Introduction}

Cyclodextrins (CD) are cyclic oligosaccharides composed of D-glucopyranose segments linked through $\alpha$-1, 4-glycosidic bonds [1]. CDs possess a toroidal shape with outer hydrophilic surface and inner hydrophobic cavity [2]. The more extensive edge of the cyclodextrins contains the secondary hydroxyl groups while the less extensive edge contains the primary hydroxyl groups. The cyclodextrin based systems improves the solubility of the drugs, increases the absorption and stability, and decreases the toxicity produced by the drugs [3]. They also ensure targeted and sustained release of drugs. Such systems are used for gene therapy, for diabetics and cholesterol related diseases, acute liver injury, cardiovascular diseases, cancer therapy etc. [4].

The interior of $\mathrm{CD}$ is hydrophobic whereas exterior is hydrophilic. Thus, through host-guest interaction, they form inclusion complex with other molecules [5]. CD form complex with either a part of the guest molecule or with the entire molecule depending on the size and properties of the guest molecule. CDs has limited solubility in water. So, in order to increase water solubility, soluble CD derivatives are prepared by reacting CDs with a variety of reagents [6]. Some of the examples of $\mathrm{CD}$ derivatives used for biomedical applications are hydroxypropylated CD [7], carboxymethylated $\mathrm{CD}$ [8], sulfobutylether CD [9] etc. CDs have insignificant toxicity, enhanced bioavailability, forms host-guest complexation in reversible manner and it also have the ability to modify the physio-chemical properties of guest molecules. Because of all these properties, CD based host-guest systems find wide application in gene and drug delivery [[10]]. Anticancer drugs can be effectively encapsulated on such system and thereby producing controlled, selfdirected and aimed release of drugs to tumor sites. When such systems are exposed to certain stimuli such as temperature, $\mathrm{pH}$, light, voltage, enzyme, redox etc. they exhibit quick physical and chemical transitions [11]. Stimuli responsiveness can be brought to a CD based system in two ways. A simple way to achieve stimuli responsiveness is through a reversible host-guest interaction between cyclodextrins and guest molecules. The other way is, CDs can be functionalized on host or guest molecules to introduce additional stimuli responsive groups through covalent conjugation or supramolecular recognition. When the stimuli responsive guest molecules interact with this host-guest system which is CD-modified, it results in the formation of CD-modified host-guest system, which is stimuli sensitive.

In this review, we focus mainly on the research advances of multiple stimuli responsive $\mathrm{CD}$ based systems and their biomedical applications in the last ten years. Such systems have wide applications in drug and gene delivery, cancer therapy, 3D cell cultures, cosmetics, electrochemical therapeutics etc.

\section{Multiple stimuli responsive CD based materials}

Tao Cai and coworkers reported a stimulus-responsive hydrogel network containing threaded $\beta$-cyclodextrinterminated chains by using a fusion of regulated radical polymerization and click chemistry [12]. The

\footnotetext{
* Corresponding author: sandhyasadanandan@am.amrita.edu
} 
synthesized hydrogel network was poly (ethylene glycol)-block-poly (propylene glycol)-block-poly (ethylene glycol) (PEG-b-PPG-b-PEG). The sliding $\beta$ cyclodextrin-capped-poly (2-(methacryloyloxy) ethyl succinate) ( $\beta$-CD-capped PMES) was then incorporated to this hydrogel network. In the presence of $\beta-C D$ terminated chains ( $\beta$-CD-capped PMES), an ultraviolet light triggered thiol-ene click reaction between a diacrylate polymer (PEG-b-PPG-b-PEG) and a crosslinking agent pentaerythritol tetrakis (3mercaptopropionate) (PETMP) was used to prepare the hydrogel. During this UV-triggered thiol-ene click reaction to form a network, there occured a host-guest interaction between $\beta$-CD-ring and the poly (propylene glycol) (PPG) portion of PEG-b-PPG-b-PEG polymer. And due to this interaction, the $\beta-C D$ terminated PMES chains get caged within the grid of this PEG-b-PPG-bPEG hydrogel's network. Through RAFT polymerization technique, $\beta$-CD-capped PMES polymer was synthesized, which contains 2 PMES segments on it and a $\beta-C D$ ring on both terminals. This polymer was $\mathrm{pH}$ sensitive to the hydrogel network of thermo-responsive PEG-b-PPG-b-PEG. Thus, under aqueous conditions, the equilibrium swelling ratio of this threaded polymer hydrogels with sliding $\beta$-CD rings (PEG-b-PPG-b-PEGthread- $\beta C D$-capped-PMES) was depended on both $\mathrm{pH}$ and temperature. Hence, they can be effectively used as biomaterials in drug transport system, tissue engineering, etc.

Qing- Lan $\mathrm{Li}$ and coworkers synthesized biocompatible nanovalves which are stimuli responsive [13]. At the top of azobenzene-functionalized mesoporous silica nanoparticles (MSNs), the poly (glycidyl methacrylate) (PGMA) polymers, which were incorporated with $\beta-C D$, were effectively assembled. It occurs through the host-guest interaction between azobenzene and $\beta-C D$ to form MSN@PGMA-CD hybrid nanoparticles. $\beta-C D$ was incorporated to PGMAs to form PGMA-CDs, by the effective addition of amine groups to it through ring opening reaction. The PGMACDs are highly biocompatible and are highly soluble in water, and can also be used as a polymer supramolecular host to form inclusion compounds with appropriate guest molecules. They have also studied about the controlled release of cargo by MSN@PGMA-CD hybrid nanoparticles, on exposure to various stimuli like UVlight, change in temperature, and competitive binding agents. By using 3-(4, 5-dimethylthiazol-2-yl)-2, 5diphenyltetrazolium bromide (MTT) as the standard, the biocompatibility of MSN@PGMA-CD hybrid nanoparticles were checked. Its minimal cytotoxicity to cells further supports its use as a drug transporter in drug delivery field. When activated by light, temperature changes and competitive binding agents, the experimental results indicated that these types of mesoporous hybrid nanocomposites can effectively encapsulate and release cargo when compared to conventional nanovalves having $\beta-\mathrm{CD}$ itself as the gated constituent. At low concentrations, they have insignificant cytotoxicity, so they can be used as nanocontainers to control drug delivery.

$\mathrm{Yu}$ Xiao and coworkers reported a regulated release system which is both voltage and enzyme responsive [14]. Here mesoporous nanoparticles (MSNs) are employed. Due to their customizable particle size, huge surface area, pore capacity, and excellent chemical and thermal stability, MSNs can be used as carriers for controlled drug release applications. Ferrocene derivatives are added to MSNs and these ferrocene $(\mathrm{Fc})$ groups get modified on the pores of MSNs, and at the opening of the pore, forming an inclusion compound with $\beta$-cyclodextrin $(\beta-\mathrm{CD})$. The pores are prevented from being opened due to size of the inclusion complex formed. The premature delivery of Rhodamine $\mathrm{B}(\mathrm{RhB})$, before stimulation, is inhibited by the inclusion complex formed. It is then stimulated by hydrogen peroxide and heme protein (horseradish peroxidase (HRP) or haemoglobin $(\mathrm{Hb})$ ), glucose oxidase (GOD) with HRP and glucose or by applying a voltage $+1.5 \mathrm{~V}$. It is observed that during stimulation, the charged ferrocenyl groups which are present in the pores of MSNs undergo rapid dissociation from the cavity. The nanovalves are opened when $\beta-C D$ exits the functionalised MSN thus releasing $\mathrm{RhB}$. The drug delivery can be improved when the release system is multi stimuli-responsive. As this system is responsive to both enzymes with glucose and voltage, it can be effectively used for the detection of glucose levels in blood, electrochemical therapeutics.

Tianxin Miao and coworkers reported alginate-based hydrogels which are both temperature and strain sensitive and are dually cross-linked and have medium mechanical characteristics [15]. They also have selfhealing ability. Alginate (Alg) is a polysaccharide derived from plants. Preparation of this hydrogel depends on the formation of a supramolecular inclusion compound between Pluronic F108 (poly (ethylene glycol)-b-poly (propylene glycol)-b-poly (ethylene glycol)) or PEG-b-PPG-b-PEG and Alginate-graft- $\beta$ $\mathrm{CD}$ (Figure 1a). When $\beta-\mathrm{CD}$ is conjugated with biological macromolecules and mixed with guest molecules, it results in the formation of a hydrogel network, which is physically cross-linked. When alginates are covalently conjugated with $\beta-C D$, the functionality of the large polymer gets improved. Because of their small cytotoxicity value and inherent temperature responsiveness, bifunctional copolymers such as PEG-b-PPG-b-PEG (Pluronic F108) can be effectively used as drug transporters in drug release systems. They can be also used for cell cultures. The purpose of this research was to prepare an alginate grafted $\beta-\mathrm{CD}$ as the host biological macromolecule and insert Pluronic F108 to it, which is the bifunctional guest molecule, to create a physically cross-linked mediumhardness hydrogel. After cross-linking, the intermolecular entanglement between the guest polymer (such as PPG) and the alginate grafted $\beta-C D$ host molecule formed a unique structure through which they can achieve shear thinning property. Pluronic F108 is 
A

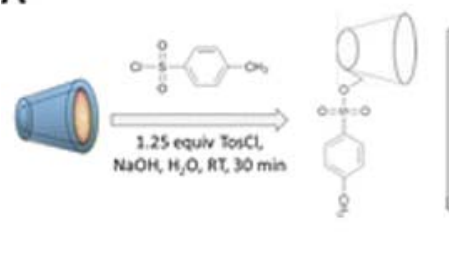

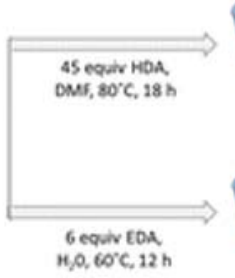
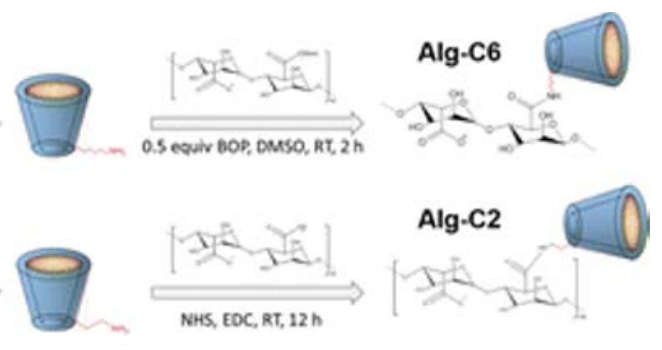

\section{B}
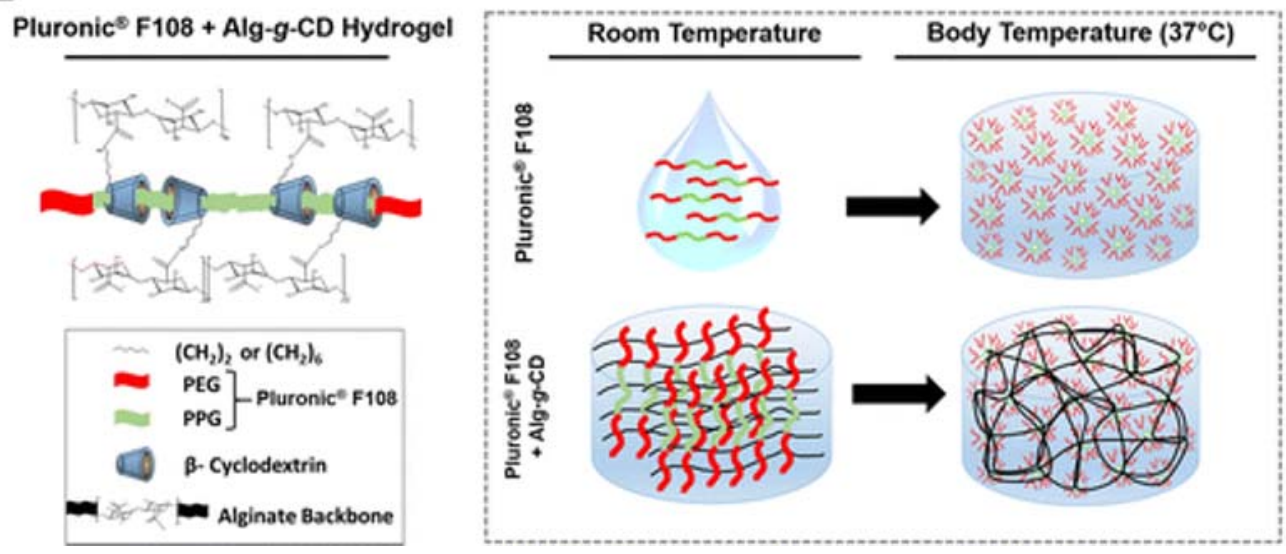

Fig. 1. (a) Schematic representation of the preparation of Alg-g-CD; (b) Schematic representation of physical crosslinking of the hydrogel and its stimuli response. Adapted with permission from ref 15. Copyright $2021 @$ American Chemical Society.

responsible for the temperature sensitive property of the hydrogel. As a result, when the body temperature is raised, a double-crosslinked hydrogel is formed (Figure 1b). The shear storage modulus of this hydrogel reached a high value of $30 \mathrm{kPa}$ at body temperature. Thus, at body temperature, the doubly -crosslinked, dualstimulus-responsive hydrogel's mechanical characteristics can be adjusted to $30 \mathrm{kPa}$, which is very beneficial for cell transplant, drug delivery system etc.

Ewelina Wajs and coworkers synthesized a redox and light responsive nanocapsules (NCs) [16]. The host molecule was cyclodextrin based polymer and guest molecule was a complementary azobenzene carriers or ferrocene. The nanocapsules were prepared based on dextran polymers with ferrocene or azobenzene group, that is, azobenzene/ $\alpha-\mathrm{CD}$ dextran polymers or $\mathrm{Fc} / \beta-\mathrm{CD}$ grafted dextran polymers. When light or chemical stimulus was applied, there occurred a variation in the stability of host-guest molecules. Thus, the permeability of nanocapsules were enhanced and the structure of the capsule wall got altered. Thus, on exposing to light or redox stimulus, the encapsulation properties of the nanocapsules were improved allowing the guest molecules to rapidly diffuse through different layers of the polymer. It was also observed that this nanocapsules have reversible encapsulation and release properties.

Xiaoe Ma and coworkers synthesized supramolecular hydrogels which are multiple stimuli ( $\mathrm{pH}$ and temperature) responsive [17]. The host and guest polymers were prepared from poly (methyl vinyl etheralt-maleic acid) (P (MVE-alt-MA)). The host molecule was $\beta$-cyclodextrin-grafted P (MVE-alt-MA) (P (MVEalt-MA)-g- $\beta$-CD) and guest molecule was azobenzenegrafted $\mathrm{P}$ (MVE-alt-MA) (P (MVE-alt-MA)-g-azo). Then the aqueous solutions of both these polymers were mixed. The host-guest complexation between azobenzene and $\beta$-cyclodextrin produced the required supramolecular hydrogel P (MVE-alt-MA)-g- $\beta-\mathrm{CD} / \mathrm{P}$ (MVE-alt-MA)-g-azo. To confirm the formation of hydrogels, the two-dimensional NMR spectroscopic method called nuclear overhauser effect spectroscopy (2D-NOESY) was performed. In addition to the $\mathrm{pH}$ and thermal response of this supramolecular hydrogel, it also exhibited good cytocompatibility. Scaffolds required for $3 \mathrm{D}$ cell culture can be effectively made from this hydrogel due to its high biocompatibility. The cells should be able to live in the hydrogel as a $3 \mathrm{D}$ cell scaffold material. Through confocal microscopy, it was checked whether the SKOV3 cells (ovarian cancer cells) were able to remain alive in different layers of the hydrogel. During the cell culture process, SKOV3 cells were active, remained alive and diffused at different depths in the supramolecular hydrogel. Thus, this hydrogel finds application in separation of cells, drug release, 3D cell cultures etc.

Yang Kang and coworkers synthesized a supramolecular micelle system which is sensitive to ROS as well as glutathione and can form stable micelles in aqueous medium [18]. $\beta$-cyclodextrin, which is present at the terminal of methoxy polyethylene glycol (mPEG- $\beta$-CD) showed non-covalent interactions with ferrocene $(\mathrm{Fc})$ adapted on camptothecin $(\mathrm{Fc}-\mathrm{CPT})$ to 
form this redox-responsive supramolecular assembly. They react to form an inclusion complex mPEG- $\beta$ $\mathrm{CD} / \mathrm{Fc}-\mathrm{CPT}$, which can make stable, homogeneous sphere-shaped micelles in water. These micelles have high drug loading ability. It is observed that these micelles can release CPT at tumor sites more rapidly than releasing it to normal cells. The concentration of glutathione (GSH) is larger in tumor sites when compared with normal cells. The tumor cells also produce high level of reactive oxygen species (for example: $\mathrm{H}_{2} \mathrm{O}_{2}$ ) than normal cells. So, when a higher level of $\mathrm{GSH}$ or $\mathrm{H}_{2} \mathrm{O}_{2}$ is present in the cytoplasm after the endocytosis process, then it would suddenly ruin the linkage between $\beta-C D$ and ferrocene. It can also break the disulphide bonds in micelles. Thus, as the chain cleaves in a regular manner, it would simultaneously result in the release of drugs to tumor cells rapidly.

Ankur A. Awasthi and Prabhat K. Singh reported an assembly of supramolecular aggregates of Auramine $\mathrm{O}$ $(\mathrm{AuO})$ which is templated by sulfated cyclodextrin [19]. This assembly is responsive to both temperature and ionic strength. Auramine $\mathrm{O}$ is an amyloid marker and is taken as the cationic guest molecule. Due to high negative charge density of sulfated derivative of $\beta-C D$ (SCD), it can promote effective aggregation of cationic guest molecules and thus it was taken as the anionic host molecule. Here in this study, AuO was reacted with SCD to form highly emissive aggregate. That is, they showed longer excited state lifetime. It was observed that the Jaggregate of the dye is responsible for the high emissive property of aggregates, more than the complex formed through host-guest interaction between $\mathrm{AuO}$ and SCD. The photophysical nature of AuO-SCD assembly was found to be similar to photophysical nature of $\mathrm{AuO}$ in human insulin fibrillar media. AuO emits strongly in a highly charged polyanionic SCD environment, with the largest red-band shift with an emission maximum at 560 $\mathrm{nm}$. AuO in human insulin fibrillar media also shows a highly emissive red-shifted band at $560 \mathrm{~nm}$. In both the cases (s AuO-SCD assembly and self-assembled AuO), the established time-resolved emission spectra (TRES) showed a strong red shift, which was an indication of transfer of excitons in the $\mathrm{AuO}$ aggregates generated. The newly synthesized assembly of supramolecular aggregates were extremely sensitive to temperature and ionic strength and can be used to construct stimulus responsive systems.

Xing Wang and coworkers reported a supramolecular hydrogel which is multi-stimuli sensitive [20]. Through the host-guest complexation between poly (ethylene glycol) polyhedral oligomeric silsesquioxane (CD)7 (PEG-POSS-(CD)7) polymer and Azo-SS-Azo dimer, this hydrogel is synthesized. This polymer has a tadpole like shape. When rigid POSS units were incorporated to the host polymer PEG, it produced polymeric network with high mechanical characteristics. Multi-stimuli responsive behavior was imparted to the host-guest molecules by the azo guest molecules, which are linked by disulfide bonds. Thus, these hydrogels are sensitive to light, temperature, reducing agent and azobenzene molecules. Temperature, light and redox may cause a reversible gel-sol phase transition in supramolecular hydrogels, and azo derivatives may cause a rapid gel-sol transition. These hydrogels are highly biocompatible and have high ability to self-heal. Thus, they find great potential in various biomedical and healthcare systems.

Xiaoyu Xu and coworkers synthesized an NIR (nearinfrared) /pH-responsive nanocomposite smart hydrogel [21]. It was synthesized by copolymerization reaction between $\mathrm{N}$-isopropylacrylamide (NIPAAm) and methacrylated $\beta$-cyclodextrin-based macromonomer (MPCD). By using the hydrophobic cavity structure of $\beta-C D$, they have created a $\beta$-CD-related linear polymer (PCD) which is water soluble, and has an outstanding capacity to make inclusion compounds with various guest molecules. This PCD is then reacted with glycidyl methacrylate (GMA) to synthesize $\beta$-cyclodextrin-based methacrylated macromonomer (MPCD). Through hostguest reaction, adamantine conjugated doxorubicin (ADDOX) prodrug was effectively loaded into the hydrogel. A hydrazone bond conjugates adamantine with DOX. This bond was labile at acidic $\mathrm{pH}$ and remained stable at physiological $\mathrm{pH}$. It was observed that at acidic $\mathrm{pH}$, that is, at $\mathrm{pH} 5.5$ and 6.5, DOX was released faster when compared to the release of DOX at $\mathrm{pH}$ 7.4. Gold nanorods (GNRs) were integrated into this hydrogel network. This is done to improve mechanical characteristics of hydrogel. In addition, the nanocomposite hydrogel containing GNR and the thermally responsive PNIPAAm network has good NIR light responsiveness. Photoresponsive hydrogels that respond to near-infrared light are of great importance as this NIR light can penetrate through human tissues with adequate intensity, thereby minimizing damage to tissues. The nanocomposite hydrogel has a very uniform three-dimensional porous structure and a constant drug release pattern, enabling it to release DOX slowly and stably for more than one month. Through the in vitro cytotoxicity test, it is observed that the nanocomposite hydrogel has suitable biocompatibility and showed good photothermal effect. In the in vivo antitumor test, it is observed that as the drug resides at the cancer cells for a longer time period and due to the effective photothermal process, this nanocomposite hydrogel has the ability to have less adverse effects in the chemo-photothermal synergistic treatment. Therefore, this nanocomposite hydrogel has broad prospects and can be widely used as a prolonged drug release system for chemophotothermal cancer treatment.

Weiju Hao and co-workers promisingly studied about dual stimuli responsive NPs for enhanced drug delivery[22]. Dual stimuli responsive NPs with $\mathrm{pH}-$ and photo- responsibility were synthesized via host-guest reaction. AZO-PDPAn-PEG45, APP (azobenzene -poly2-(diisopropylamino) ethyl methacrylate - methoxy polyethylene glycols) was a self-synthesized copolymer that can act as a guest and also, it can be inserted into the cyclodextrin (host). In this work they demonstrated the 
efficiency of $\mathrm{pH} /$ photo dual-sensitive nanoparticle (UCNP-CD@APP) in drug delivery. These NPs were found to be biocompatible, controllable release of drug, efficient intracellular delivery and tumor targeting.

A supramolecular nanoparticles assembly having Poly ( $\alpha$-cyclodextrin) as the host molecule and azobenzene linked acetal-modified $\beta$-cyclodextrin as the guest molecule which are dual stimuli sensitive was synthesized by Zhang et. al [23]. Azobenzene is UV responsive and acetal is $\mathrm{pH}$ responsive. To check the performance of drug carriers for cancer therapy, in vitro cytotoxicity test was conducted and it revealed that the supramolecular polymer nanoparticles were non-toxic in nature. The $\mathrm{pH}$ sensitiveness of these polymeric nanoparticles is beneficial for the regulated delivery of drugs as tumor tissues are more acidic (pH 5.0) when compared to normal physiological cells ( $\mathrm{pH} 7.4)$. When irradiated with UV light, the drugs loaded in this polymeric nanoparticle were effectively released to the targeted tumor tissues at a controlled rate.

Glutathione/pH-responsive nano sponges $(\mathrm{sS})$ were used to influence strigolactone release to prostate tumor cites in another interesting study [24]. This assay of dual stimuli responsive nano sponges has great impact in the most challenging areas of medical field. Mainly, doxorubicin-carrying glutathione targeted nano sponges showed high influence against tumor cites with high glutathione concentration. These novel nano sponges responsive to both $\mathrm{pH}$ and redox stimuli have high capacity for targeted drug delivery. They can release two Strigolactones (SL) analogues for curing cancer disease and moreover it has the capacity to deliver this Ns with spatio-temporal control. These SLs are plant hormones (carotenoid-derived) that show anti-tumor activities. They synthesized SL-loaded glutathione/pH-responsive $\mathrm{Ns}$ (GSH/pH-NS) to particularly release SLs to influence their therapeutic activity and prostate cancer cells.

Yang Bai and coworkers synthesised supramolecular prodrug complexes (SPCs) which are both photo and $\mathrm{pH}$ responsive [25]. $\beta$-cyclodextrin-acylhydrazone-DOX and poly (2-(dimethylamino) ethyl methacrylate) with azobenzene terminal (Azo-PDMA-FA) were used as the building units. On exposure to UV-light, firstly, the SPCs self-assembled to form multicompartment vesicles and then changed to complex micelles. It was found that the obtained SPCs based complex micelles and multicompartment vesicles have fine reversibility when irradiated with ultraviolet and visible light alternatively. In addition, using UV and $\mathrm{pH}$ stimulation, a three-stage organized drug delivery property was exhibited by the dual stimuli sensitive SPCs-based self-assemblies. DOX was released in a slow rate, when SPC initially selfassembled to form multi-compartment vesicles. There occurred a transition in morphology due to dissociation of $\beta-C D / A z o$, induced by UV-irradiation and then DOX was released in an enhanced manner. The acylhydrazone bonds were broken as the self-assemblies passed on to PBS solution of $\mathrm{pH} 5$. A comparatively high increase in DOX release was then observed. Since the acylhydrazone bond was hydrolysed to a certain extent under a $\mathrm{pH}$ condition of 7.4, then under the absence of any stimuli, small quantity of DOX was separated from $\beta$-CD. Subsequently, UV irradiation transforms the multicomponent vesicles into complex micelles with larger sizes. Thus, DOX which got hydrolyzed from $\beta$ $\mathrm{CD}$ showed an increased rate of diffusion. Finally, due to the $\mathrm{pH}$-sensitive acylhydrazone bond, DOX was rapidly separated from $\beta-\mathrm{CD}$ after $\mathrm{pH}$ changes in PBS. As a result, SPC-related self-assemblies with both UV response and $\mathrm{pH}$ response capabilities can achieve programmed drug release.

Arpita Roy and coworkers synthesized a biopolymeric hydrogel which is both temperature and $\mathrm{pH}$ responsive [26]. Here a dual stimuli sensitive $\beta-\mathrm{CD}$ based hydrogel was synthesised for the delivery of colonic drugs such as metronidazole and ofloxacin to the targeted cells. Poly (N-isopropyl acrylamide) (PNIPAm) and poly (methacrylic acid) (PMAc) were crosslinked and grafted on $\beta-\mathrm{CD}$ to form $\beta$-CD-cl-(PNIPAm-coPMAc) hydrogel. This hydrogel showed high temperature and $\mathrm{pH}$ sensitiveness, as they contain PNIPAm and PMAc units in it. It was sufficiently stable. It can simultaneously transport metronidazole and ofloxacin in a controlled manner due to its porous structure. Based on swelling parameters, it was observed that the hydrogel is dual stimuli responsive (temperature and $\mathrm{pH})$. The hydrogel network appeared to be noncytotoxic to MG-63 cell lines. The gel released metronidazole and ofloxacin simultaneously in vitro, indicating that it releases two colonic drugs at the required $\mathrm{pH}$ (colon $\mathrm{pH}$ 7.4) and body temperature $\left(37^{\circ} \mathrm{C}\right)$.

Multifunctional nanotheranostics which are $\mathrm{pH}$ and temperature responsive were reported by Manita Das and coworkers [27]. For dual drug delivery, multifunctional nanoconjugates with multiple basic functions (such as magnetism, fluorescence, cell targeting, $\mathrm{pH}$ and thermal response characteristics) have been produced. N-isopropyl acrylamide (NIPAM) and $\beta$ Cyclodextrin modified with maleic anhydride combined to form the required CD-MA-NIPAM copolymer. As this nanocarrier is both $\mathrm{pH}$ and thermo responsive, they can release the drug when subjected to a change in temperature and $\mathrm{pH}$. To check the multiple delivery of drugs by nanoconjugates, the model drugs, curcumin and DOX were used. As this nanoconjugates contain cyclodextrin, they can actively load and deliver drugs which were both hydrophobic and hydrophilic. DOX is hydrophilic in nature and curcumin is hydrophobic in nature. Both curcumin and DOX can be simultaneously loaded and released by this nanoconjugates. Curcumin is a low bioavailable drug and it was found that this nanoconjugates were able to improve the endocytosis of curcumin. It was observed that at elevated temperature, drug release took place at a faster rate for both DOX and curcumin due to thermosensitive property of PNIPAM. Hence these dual stimuli responsive nanoconjugates can be effectively used for cancer therapy. 


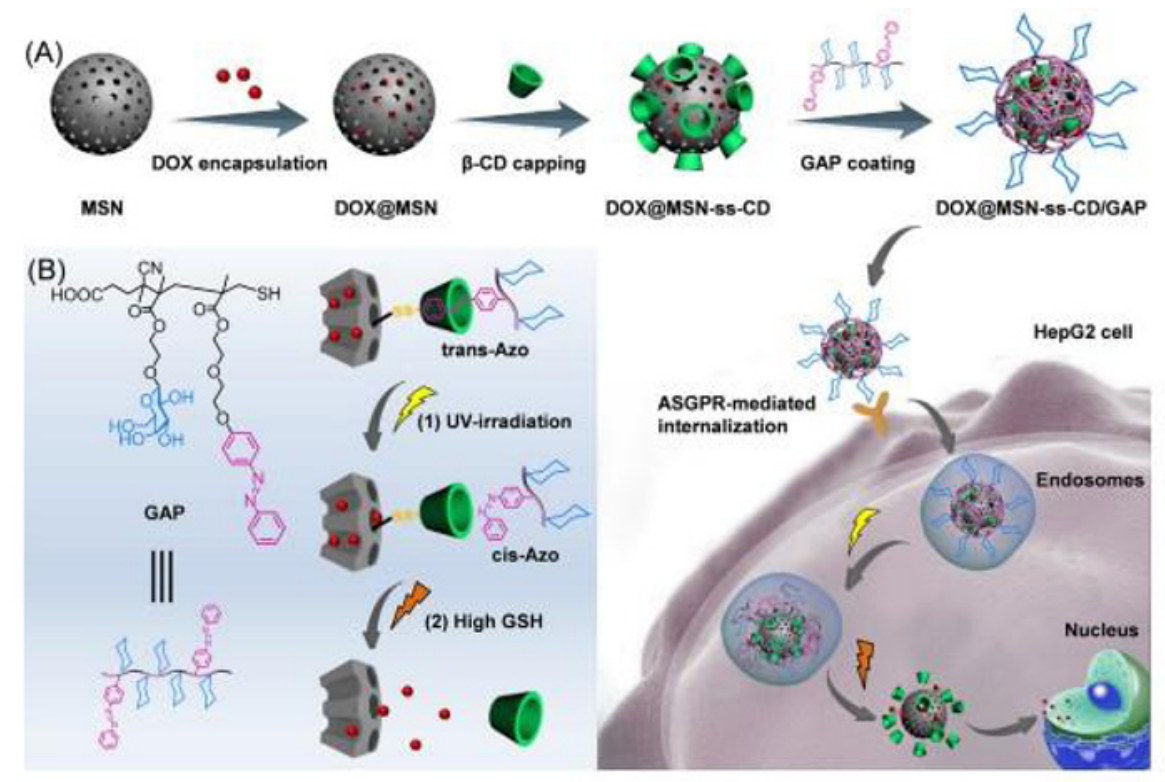

Fig. 2. (a) Schematic representation of the formation of cyclodextrin-based mesoporous silica nanoparticles ( $\beta$-CD-g-MSNs). (b) Schematic representation of their UV and GSH responsive DOX delivery. Reproduced with permission from Elsevier.

Jingwei Wang et al. synthesized another dual stimuli sensitive drug carrier $\beta$-CD (CS-g- $\beta-C D)$ [28]. The CSg- $\beta-C D$ with a three-dimensional (3D) porous structure allowed the polar functional groups (amino and hydroxyl) to be completely exposed on the surface. After characterization of CS-g- $\beta-\mathrm{CD}$, an anticancer drug etoposide (VP16) was incorporated to it. The kinetic release studies of VP16 indicated that the delivery of VP16 from VP16-incorporated CS-g- $\beta$-CD depends on $\mathrm{pH}$ as well as temperature.

Yaling $\mathrm{Wu}$ and coworkers synthesized dual stimuli responsive cyclodextrin-based mesoporous silica nanoparticles ( $\beta$-CD-g-MSNs) for anticancer treatment [29]. Azobenzene is highly photosensitive and in trans configuration it can readily enter into the cavity of cyclodextrin through van der Waals forces and hydrophobic interaction and thus can form complexes. But cis-azobenzene can't form complexes with $\beta-C D$, as its structure is not in match with structure of $\beta-C D$. Upon UV irradiation, the configuration of azo varies from trans to cis and upon visible light irradiation, azo changes from cis to trans. So, when the supramolecular assembly is alternatively irradiated with UV and Visible light, the azo units present in it get self-assemble and segregate reversibly. Also, through a redox-sensitive disulfide bond, the release rate of DOX is limited by $\beta$ CD grafted MSN, which act as a gate keeper. $\beta$ - CD grafted MSN act as the primary gate keeper and azobenzene/galactose grafted polymer (GAP) act as the secondary gate keeper to stop the flow of molecules from inside. When irradiated with ultraviolet radiation, there occurs the decomposition of the azobenzene part from the $\beta-C D$ cage resulting in controlled and regulated release of DOX. It is followed by release of glutathione (GSH). Then GSH molecules will break the disulfide bond and detachment of $\beta-C D$ takes place, which results in the release of drugs in a rapid manner. The redoxsensitive release of DOX was verified by in vitro studies. It was also found that, when compared to HeLa and COS7 cells, the DOX encapsulated MSN-ss-CD/GAP (DOX@MSN-ss-CD/GAP) was able to transport DOX more effectively to HepG2 cells (Figure 2b).

Panjun Wang and coworkers synthesized graphene oxide (GO) hybrid supramolecular hydrogels which are NIR (Near Infrared), temperature and $\mathrm{pH}$ responsive [30]. A quaternization reaction occured between $\mathrm{pH}$ responsive poly (N, N-dimethylaminoethyl methacrylate) (PDMAEMA) and poly (ethylene glycol) monomethyl ether with a bromine terminal (mPEG-Br). When the temperature rised to a certain level, the supramolecular hydrogel formed by the host-guest interaction of poly (ethylene glycol) monomethyl ether (mPEG) and $\alpha$-cyclodextrin transformed into a mobile sol phase. To give further crosslinking, graphene oxide sheets were used as the core material. GO interacts with mPEG-QPDMAEMA through an electrostatic selfassembly to produce the required GO hybrid cyclodextrin based supramolecular hydrogel. In addition to this, GO also absorbs near-infrared light and converted it into heat, thereby triggering the sol-gel transition. GO also imparted thermal stability to this hydrogel. The prepared hydrogels contain GO which was NIR sensitive, host-guest complex which was thermosensitive and PDMAEMA which was $\mathrm{pH}$ sensitive. As this hydrogel responds to multiple stimuli, it can be effectively used for controlled cargo delivery.

Hai Yao and co-workers reported a triple-stimuli responsive supramolecular self-assembly for the sustained release of DOX [31]. The initial spherical selfassemblies (ISSA) were formed from $\beta$-CD dimers (Fc- 
CD2) which was generated by linking $\beta$-CD which with ferrocene (Figure 3a). This ISSA showed sensitiveness towards both ultrasound and redox. The ISSA was made into larger spherical self-assemblies (LSSA) by inserting polyethylene glycol (PEG) functionalized with benzimidazole (BM) on one side and lactobionic acid (LA) on the other side on to the exterior of ISSA. The BM-PEG-LA was inserted onto the ISSA surface through the host -guest interconnection between $\beta-C D$ and BM. The $\beta$-CD-P-SNO interconnection showed sensitivity towards acidic $\mathrm{pH}$ and also ensured targeted drug release. Once the LSSA entered the acidic tumor environment the BM-PEG-LA unit got separated from the surface of LSSA because of the disintegration of BM- $\beta$-CD interconnection. The LSSA after the separation of BM-PEG-LA, when subjected to ultrasound sound formed branched aggregates (BA) as the host-guest interconnection between $\mathrm{Fc}$ and $\beta-\mathrm{CD}$ got strengthened. In the presence of these $\mathrm{H}_{2} \mathrm{O}_{2}$ these BA got disintegrated and formed secondary spherical selfassemblies (SSSAs). This triple-stimuli mediated transformation from LSSAs to SSAs was utilized for drug delivery. The $\mathrm{pH}$ stimulated drug release behavior was studied by exposing the DOX-loaded LSSAs to two different $\mathrm{pH}$ environments of 7.4 and 6.0. A greater drug release was observed when the LSSAs was subjected to pH 6.0 indicating acid stimulated drug release. The drug release increased more rapidly when it was subjected to ultrasound followed by treatment with $\mathrm{H}_{2} \mathrm{O}_{2}$ after $\mathrm{pH}$ treatment (6.0) (Figure 3b). The LSSAs also showed good biocompatibility, sustained drug release and also enhanced cellular intake.

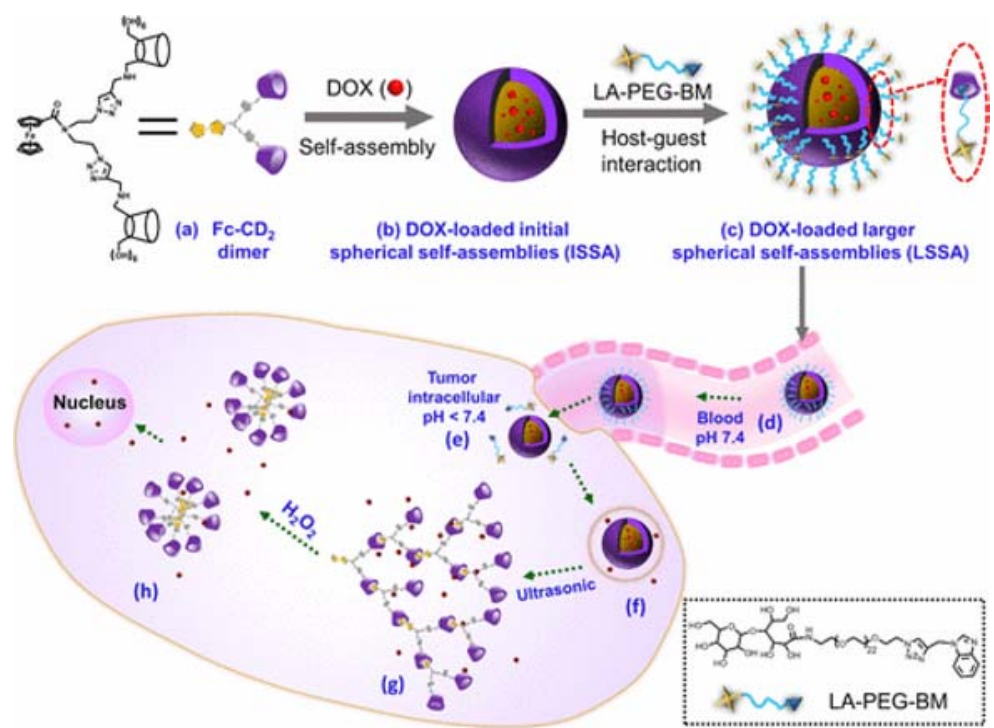

Fig. 3. (a) Schematic representation of the formation of the speherical nanoassemblies. (b) Schematic representation of their $\mathrm{pH}$, $\mathrm{H}_{2} \mathrm{O}_{2}$ and ultrasound responsive DOX delivery. Adapted with permission from ref 31. Copyright $2021 @$,American Chemical Society.

Feng Guo and co-workers had constructed a double stimulus -sensitive nanocarriers by gathering mesoporous silica nanoparticles enhanced with 2mercaptobenzimidazole (MSN-BM) and star polymer consisting of poly( $\mathrm{N}$-isopropylacrylamide) together with a $\beta$-CD nucleus $(\beta$-CD-(PNIPAM)7) [32]. The nanocarriers was formed because of host-guest interconnection between $\beta-\mathrm{CD}$ and BM. The $\beta-\mathrm{CD}-$ (PNIPAM)7@MSN-BM nanocarriers exhibited a wellorganized mesoporous structure, which was observed from the TEM image. The DOX drug was embedded into the channels of MSN via the electrostatic attraction between the positively charged DOX and MSNs and it was blocked from escaping via host-guest interconnection between $\beta-\mathrm{CD}$ and BM. Thus, $\beta-\mathrm{CD}$ (PNIPAM) 7 acted as a doorkeeper, managing DOX release. It was seen that a larger amount of DOX was escaped from the nanocarrier when the $\mathrm{pH}$ was reduced from 7.0 to 3.0. The elevated drug release at $\mathrm{pH} 7.4$ was attributed to the disintegration of host-guest interconnection between $\beta-\mathrm{CD}$ and $\mathrm{BM}$. The temperature-sensitive drug release of nanocarrier was contributed by PNIPAM polymer. When the temperature was raised above the lower critical solution temperature of the polymer, it exhibited a hydrophobic behavior in water which led to the destruction of the hydrated structure of PNIPAM enabling drug release. These nano carriers were mainly used for delivering tumor medications.

JianGuo Zhang and coworkers synthesized dual stimuli sensitive supramolecular assemblies for effective drug delivery [33]. It was prepared via the host-guest complex formation between $\beta$-CD-graft-poly (2(dimethylamino) ethyl methacrylate) ( $\beta-\mathrm{CD}-\mathrm{g}-$ PDMAEMA) (host polymer) and poly ( $\varepsilon$-caprolactone) modified with azobenzene (Azo-PCL) (guest polymer). Upon exposure to uv-visible irradiation and at different $\mathrm{pH}$ conditions, the assembly which had a uniform spherical shape changed to irregular aggregates. Photosensitive azobenzene underwent a cis-trans 
isomerization in presence of light. In trans configuration they readily enter into the cavity of cyclodextrin through van der Waals forces and hydrophobic interaction. Upon UV irradiation, the configuration of azo changes from trans to cis and upon visible light irradiation, azo changes from cis to trans. So, when the supramolecular assembly is alternatively irradiated with uv and visible light, the azo units present in it get self-assemble and segregate reversibly. The size and morphology of micelles uniformly vary with the ratio of host /guest molecules and $\mathrm{pH}$ nature. PCL is hydrophobic and formed the core of the micelle, and PDMAEMA, which is hydrophilic, was used as the shell. It can thus sense $\mathrm{pH}$ variations. The blank supramolecular assemblies were found to be nontoxic through cytotoxicity assessment. To study the drug loading ability of this supramolecular micelle, DOX was loaded to it. And it was observed that the drug-loaded micelles showed effective anticancer activity and hindered the proliferation of cancer cells. The drug was released to cancer cells at a faster rate when it is subjected to an acidic $\mathrm{pH}$ and continuously exposed to UV-Visible light.

Lin Yin-Ku and coworkers reported $\beta-\mathrm{CD}$ based ferrocene supramolecules which are both photo and redox sensitive [34]. Methoxy polyethylene glycol (MPEG) was conjugated with ferrocene $(\mathrm{Fc})$ terminal to form MPEG-Fc, which is redox sensitive. $\beta-C D$ and poly (4-substituted- - $\varepsilon$-caprolactone) (PXCLn) are connected by a photocleavable 5-hydroxy-2-nitrobenzyl alcohol (ONB) to form $\beta$-CD-ONB-PXCLn, which is photodegradable. A host-guest complex formation occured between MPEG-Fc and $\beta$-CD-ONB-PXCLn, resulting in the formation of supramolecular complex, which was both redox and photo sensitive. The inclusion complex thus formed was MPEG-Fc/ $\beta-C D-O N B-$ PXCLn. In aqueous medium, this complex can form micelles and these micelles were found to be spherical in structure. DOX was incorporated to this micelle complex to check its drug loading ability. In vitro cytotoxicity test indicated that these complex micelles are highly biocompatible. The complex micelles decomposed and effectively delivered DOX when treated with hydrogen peroxide and irradiated with UVlight.

Manita Das and co-workers introduced a nanocarrier by using carbon nanotubes (CNTs) and a CD copolymer that could respond to both temperature and $\mathrm{pH}$ [35]. This nanocarrier could provide a combined effect of photothermal therapy (PTT), cocktail chemotherapy (CCT) and angiogenesis inhibition. Cyclodextrin-maleic anhydride- N-isopropylacrylamide (CD-MA-NIPAM), a $\mathrm{CD}$ polymer with temperature and $\mathrm{pH}$ sensitivity was conjugated to CNTs whose surface was covered with carboxyl groups to form $\mathrm{CD} / \mathrm{CNT}$ nanocarriers. These nanocarriers acted as dual dug carriers by delivering DOX and curcumin. At physiological pH 7.4, DOX was accommodated in the nanosystem via electrostatic attraction between DOX protons and carboxyl groups of nanocarrier. The protonation of the carboxyl groups at acidic $\mathrm{pH}$, caused the shrinkage of the nanosystem thereby releasing the drug. Unlike DOX, curcumin was released from the nanosystem at $\mathrm{pH}$ 7.4. The curcumin was loaded on to the nanocarrier via host-guest interconnection between $\mathrm{CD}$ and curcumin. This interaction reduced the tightness of the $\mathrm{CD}$ ring thereby releasing curcumin. Also increase in the concentration of COO- ions at $\mathrm{pH} 7.4$ caused the expansion of the nanocarrier, resulting in the diffusion of curcumin out of the nanocarrier. Apart from $\mathrm{pH}$ sensitive drug release, $\mathrm{CD} / \mathrm{CNT}$ also showed temperature-sensitive drug release because of the presence of the copolymer CDPM-NIPAM. When the temperature was raised above the lower critical solution temperature of the polymer, the hydrated structure of the polymer broke down leading to the release of both DOX and curcumin. This carrier also displayed photothermal effect. As this nanocarriers possessed the ability to produce photothermal effect, the expected therapeutic effect could be achieved using minimum amount of drug. This helped to remove the toxic effects caused by the drugs. This carrier exhibited good cytocompatibility. The selected drug pair also showed enhanced cocktail effect. These dug pairs also possessed antiangiogenic effect as they subdued the expression of FGF2 and VEGF gene which promoted angiogenesis.

Temperature and $\mathrm{H}_{2} \mathrm{O}_{2}$ dual-responsive nano-valves were prepared from $\beta$-cyclodextrin-poly $(\mathrm{N}$ isopropylacrylamide) $\quad(\beta$-CD-PNIPAM)-ferrocene nanovalves modified MSNs (MSN-Fc) [36]. Ferrocene (Fc) has a sandwiched structure and high biocompatibility and in the presence of $\mathrm{H}_{2} \mathrm{O}_{2}$ it gets inserted to $\beta-C D$. The stable composite NPs would be disturbed by the addition of $\mathrm{H}_{2} \mathrm{O}_{2}$ or elevating temperatures above the lower critical solution temperature of PNIPAM. The limitations of pore channels, contraction of PNIPAM and departure of cyclodextrins segments determined the drug delivery from composite NPs. In vitro cell-growth hindrance analysis suggested this material can be used as an anticancer drug-carriers.

Yanqin $\mathrm{Xu}$ and team demonstrated the efficiency of $\mathrm{pH}$ and redox responsive MSN-S-S-CS (mesoporous silica nanoparticles -sulfur-chitosan) DDS [37]. This system was synthesized by the amide reaction of the dithiodipropionic acid by the amino group on the surface of CS and MSN. This particular drug delivery system was modified on the basis of microenvironment differences between tumor and normal tissue cells compiled by the $\mathrm{pH}$ sensitivity of $\mathrm{CS}$, and the disulfide bonds' redox responsiveness.

Khadijeh Soleimani et al. reported a $\beta-C D$ based magnetic drug release system ( $\beta$-CD-g$\left.\left.(\mathrm{PEtOx})_{7}\right) / \mathrm{Fe}_{3} \mathrm{O}_{4}\right)$ which is both $\mathrm{pH}$ and reduction responsive [38]. The MTT method was used to test the antitumor activity of Dox-loaded magnetic nanohydrogel on MCF7 cells, and then the findings were checked with the antitumor activity of free Dox as a standard. At a pH of 7.4 and at a temperature of $37^{\circ} \mathrm{C}$, the 
DOX loaded nanohydrogel released drugs at a very slow rate. When small quantity of glutathione was added to it, it was observed that the DOX release rate got increased. But when the $\mathrm{pH}$ of the medium was lowered to 5.3, the release rate of DOX showed a remarkable increase. When small quantity of glutathione was added to it at the same $\mathrm{pH}$, it indicated a high release rate of the drug. This nanohydrogel was found to be highly biocompatible.

Sedigheh Borandeh and coworkers synthesized a $\mathrm{pH}$ and redox dual stimuli $\beta-\mathrm{CD}$ sensitive supramolecular system for DOX delivery [39]. Using cystamine, graphene oxide $(\mathrm{GO})$ was modified with $\beta-\mathrm{CD}$. Through host-guest complexation of $\beta-\mathrm{CD}$ and polyethylene glycol with adamantine terminal, it was PEGylated. Thus, the nanocarrier, GO-Cys-CD-PEG was synthesized. To increase the solubility and stability and to decrease the toxicity of graphene, polyethylene glycol was used. Under physiological circumstances, there was a possibility of outflow of the drug loaded in the nanocarrier. It was prevented by using PEG. The cystamine group imparted a reduction responsive behavior to the nanocarrier. The drug release properties of DOX encapsulated GO-Cys-CD-PEG was observed in various $\mathrm{pH}$ solutions with or without the presence of glutathione. It was observed that large quantities of DOX were released from the nanocarrier at a temperature of $37 \mathrm{C}$ and at a $\mathrm{pH}$ of 5.3, with the addition of small amount of glutathione. This indicated that the drug release property of this nanocarrier is both reduction and $\mathrm{pH}$ responsive. In HepG2 cells, DOX encapsulated GOCys-CD-PEG showed greater cytotoxicity when compared to free DOX. This was due to high stability of PEG and improved dispersibility of DOX encapsulated GO-Cys-CD-PEG in the culture path.

A multiple stimuli responsive supramolecular hydrogel was synthesized by Yan's group [40]. The mAzo group in methoxy-azobenzene-grafted poly (acrylic acid) (PAA-mAzo) showed responsiveness to visible light and PAA group showed responsiveness to $\mathrm{pH}$. The hostguest complexes formed due to interaction between $\beta$ $\mathrm{CD}$ and $\mathrm{mAzo}$ moieties associates and dissociates when exposed to green and blue light. As a result of this, a solgel transition occurred reversibly. When exposed to temperature, light (visible) and $\mathrm{pH}$, the hydrogel underwent sol-gel transitions in a reversible manner. At a lower $\mathrm{pH}$, the carboxylic groups which were protonated, formed both intermolecular and intramolecular hydrogen bonds. As a result of this, PAA became sparingly soluble in water, that is, solubility decreases. Thus, it led to precipitation. The deprotonated carboxylic groups had electrostatic interactions at higher $\mathrm{pH}$ values. Thus, solubility increased when the $\mathrm{pH}$ was increased. This hydrogel acted as a suitable medium for encapsulating and delivering small molecules in a regulated manner when exposed to two stimuli. The hydrogel was loaded with rhodamine-B to check drug loading and releasing ability of this hydrogel. And it was observed that, when irradiated with green light or when exposed to a low $\mathrm{pH}$ medium, the hydrogel was able to release rhodamine-B in a controlled manner.

\section{Conclusion and future perspectives}

Here the research developments happened on multiple stimuli responsive cyclodextrin-based systems in the last decade are reviewed. The cyclodextrin system can be made stimuli responsive either through host-guest complexation, which arises due to the interaction between host and guest molecules or by adding stimuli sensitive moieties to the system through covalent conjugation or through supramolecular recognition. The CD-based stimuli responsive system can be changed into micelles, nanoparticles, hollow spheres, hydrogels etc. In vitro cytotoxicity study revealed that these CDbased stimuli sensitive systems are highly biocompatible. Various anticancer model drugs such as paclitaxel, doxorubicin, curcumin etc. are encapsulated to the system and these systems can release the drugs to targeted tumor sites under different stimuli. Thus, such systems have wide applications in drug and gene delivery, cancer therapy, 3D cell cultures, cosmetics, electrochemical therapeutics etc.

Looking into the research developments happened in the last decade it is also important to note that the research on multi stimuli responsive CD-based drug carrier is still in its infancy. Most of the reported works are pilot projects and their in vivo performances are yet to be verified. Clinical trials of most of the reported drug delivery systems are not performed. Use of these CD based these smart carriers will definitely solve many major issues in drug and gene delivery but further modification on use of desirable stimuli, drug loading efficiency, drug release time, their side effects in the body are areas yet to be explored in detail.

\section{References}

1. J. Y. Liu, X. Zhang, and B. R. Tian, Turkish Journal of Chemistry 44, 261 (2020)

2. A. Harada, Accounts of Chemical Research 34, 456 (2001)

3. B. Tian, Y. Liu, and J. Liu, Carbohydrate Polymers 242, (2020)

4. B. Tian and J. Liu, New Journal of Chemistry 44, 9137 (2020)

5. B. Tian, D. Xiao, T. Hei, R. Ping, S. Hua, and J. Liu, Polymer International 69, 597 (2020)

6. P. Saokham, C. Muankaew, P. Jansook, and T. Loftsson, Molecules 23, (2018)

7. S. Gould and R. C. Scott, Food and Chemical Toxicology 43, 1451 (2005)

8. H. Pu, P. Tang, L. Zhao, Q. Sun, Y. Zhai, Z. Li, N. Gan, Y. Liu, X. Ren, and H. Li, RSC Advances 10, 20905 (2020) 
9. V. J. Stella and R. A. Rajewski, International Journal of Pharmaceutics 583, 119396 (2020)

10. C. Muankaew and T. Loftsson, Basic and Clinical Pharmacology and Toxicology 122, 46 (2018)

11. J. Wang, Z. Guo, J. Xiong, D. Wu, S. Li, Y. Tao, Y. Qin, and Y. Kong, International Journal of Biological Macromolecules 125, 941 (2019)

12. T. Cai, W. J. Yang, Z. Zhang, X. Zhu, K. G. Neoh, and E. T. Kang, Soft Matter 8, 5612 (2012)

13. Q. L. Li, L. Wang, X. L. Qiu, Y. L. Sun, P. X. Wang, Y. Liu, F. Li, A. di Qi, H. Gao, and Y. W. Yang, Polymer Chemistry 5, 3389 (2014)

14. Y. Xiao, T. Wang, Y. Cao, X. Wang, Y. Zhang, Y. Liu, and Q. Huo, Dalton Transactions 44, 4355 (2015)

15. T. Miao, S. L. Fenn, P. N. Charron, and R. A. Oldinski, Biomacromolecules 16, 3740 (2015)

16. E. Wajs, T. T. Nielsen, K. L. Larsen, and A. Fragoso, Nano Research 9, 2070 (2016)

17. X. Ma, N. Zhou, T. Zhang, Z. Guo, W. Hu, C. Zhu, D. Ma, and N. Gu, RSC Advances 6, 13129 (2016)

18. Y. Kang, X. Ju, L. S. Ding, S. Zhang, and B. J. Li, ACS Applied Materials and Interfaces 9, 4475 (2017)

19. A. A. Awasthi and P. K. Singh, Journal of Physical Chemistry B 121, 6208 (2017)

20. X. Wang, J. Wang, Y. Yang, F. Yang, and D. Wu, Polymer Chemistry 8, 3901 (2017)

21. X. Xu, Z. Huang, Z. Huang, X. Zhang, S. He, X. Sun, Y. Shen, M. Yan, and C. Zhao, Injectable, NIR/PH-Responsive Nanocomposite Hydrogel as Long-Acting Implant for ChemoPhotothermal Synergistic Cancer Therapy (2017)

22. W. Hao, D. Liu, Y. Wang, X. Han, S. Xu, and H. Liu, Colloids and Surfaces A: Physicochemical and Engineering Aspects 537, 446 (2018)

23. Y. Dai and X. Zhang, Journal of Polymer Research 25, 1 (2018)

24. M. Argenziano, C. Lombardi, B. Ferrara, F. Trotta, F. Caldera, M. Blangetti, H. Koltai, Y. Kapulnik, R. Yarden, L. Gigliotti, U. Dianzani, C. Dianzani, C. Prandi, and R. Cavalli, Oncotarget 35813 Www.Oncotarget.Com Glutathione/PH-Responsive Nanosponges Enhance Strigolactone Delivery to Prostate Cancer Cells (2018)

25. Y. Bai, C. P. Liu, X. Song, L. Zhuo, H. Bu, and W. Tian, Chemistry - An Asian Journal 13, 3903 (2018)

26. A. Roy, P. P. Maity, A. Bose, S. Dhara, and S. Pal, Materials Chemistry Frontiers 3, 385 (2019)

27. M. Das, A. Solanki, A. Joshi, R. Devkar, S. Seshadri, and S. Thakore, Carbohydrate Polymers 206, 694 (2019)

28. J. Wang, Z. Guo, J. Xiong, D. Wu, S. Li, Y. Tao, Y. Qin, and Y. Kong, International
Journal of Biological Macromolecules 125, 941 (2019)

29. Y. Wu, Z. Xu, W. Sun, Y. Yang, H. Jin, L. Qiu, J. Chen, and J. Chen, Materials Science and Engineering C 103, (2019)

30. P. Wang, C. Huang, Y. Xing, W. Fang, J. Ren, $\mathrm{H}$. Yu, and $\mathrm{G}$. Wang, Langmuir 35, 1021 (2019)

31. H. Yao, T. Yang, J. He, G. Du, X. Song, Y. Zhang, and W. Tian, Langmuir (2019)

32. F. Guo, G. Li, S. Ma, H. Zhou, and X. Yu, International Journal of Polymeric Materials and Polymeric Biomaterials 69, 1178 (2020)

33. J. Zhang, Z. H. Zhou, L. Li, Y. L. Luo, F. Xu, and Y. Chen, Molecular Pharmaceutics 17, $1100(2020)$

34. L. Yin-Ku, W. Shiu-Wei, and L. Ren-Shen, Journal of Macromolecular Science, Part A: Pure and Applied Chemistry 58, 8 (2020)

35. M. Das, P. Nariya, A. Joshi, A. Vohra, R. Devkar, S. Seshadri, and S. Thakore, Carbohydrate Polymers 247, (2020)

36. F. Guo, G. Li, H. Zhou, S. Ma, L. Guo, and X. Liu, Colloids and Surfaces B: Biointerfaces 187, (2020)

37. Y. Xu, L. Xiao, Y. Chang, Y. Cao, C. Chen, and D. Wang, Materials 13, (2020)

38. K. Soleimani, E. Arkan, H. Derakhshankhah, B. Haghshenas, R. Jahanban-Esfahlan, and M. Jaymand, Carbohydrate Polymers 252, (2021)

39. S. Borandeh, H. Hosseinbeigi, S. S. Abolmaali, M. Monajati, and A. M. Tamaddon, Journal of Drug Delivery Science and Technology 63, (2021)

40. H. Yan, Q. Jiang, J. Wang, S. Cao, Y. Qiu, H. Wang, Y. Liao, and X. Xie, Polymer 221, (2021) 